\title{
THE MACROBENTHIC ECOLOGY OF THE STRAITS OF MAGELLAN AND THE BEAGLE CHANNEL
}

\section{ECOLOGÍA MACROBENTÓNICA DEL ESTRECHO DE MAGALLANES Y CANAL BEAGLE}

\author{
Sven Thatje \& Alastair Brown
}

\begin{abstract}
The macrobenthic community of the Straits of Magellan and the Beagle Channel was investigated using a Reineck box corer at 22 stations during the Chilean "Cimar Fiordo 3" expedition in 1997. A total of 173 taxa represented by 2188 individuals were identified and are reported for the investigated area. Clear exponential relationships with depth were revealed by analysis of abundance, biomass, species richness, and evenness. These patterns coincide with posited theories of pelagic-benthic coupling and the source-sink hypothesis of colonisation-extinction dynamics. Polychaeta dominated macrobenthic community abundance and biomass, $67 \%$ and $38 \%$ respectively, therefore consideration of biogeographic affinities concentrated on this taxon. 13 species of polychaetes observed in the study area co-occur in Antarctica suggesting biogeographic or evolutionary affinities between these adjacent regions.
\end{abstract}

Key words: biogeography, diversity, polychaete, Antarctica, Subantarctic

\section{RESUMEN}

En octubre 1997 se investigaron los ensambles macrozoobentónicos del estrecho de Magallanes y el canal Beagle con un "Reineck Box corer" en 22 estaciones durante la campaña chilena "Cimar Fiordo 3". Se identificó un total de 173 taxones representados por 2188 especímenes en el area de investigacion. Se detectó una relación exponencial de profundidad dada por el análisis de abundancia, riqueza de especies y uniformidad. Estos patrones coinciden con teorías establecidas de flujos bento-pelágicos y las hipótesis de "source-sink". Los poliquetos dominan los ensambles macrobentónicos en abundancia y biomasa en $67 \%$ y 38\% respectivamente, y por tanto el análisis biogeográfico presentado se basa en ellos. Trece de las especies de poliquetos identificados en el area investigada se conocen también por estar distribuidos en Antártica. Proponemos entonces afinidades biogeográficas y evolutivas entre ambas regiones.

Palabras clave: biogeografía, diversidad, poliqueto, Antártica, Subantártica

1 National Oceanography Centre, Southampton, School of Ocean and Earth Science, University of Southampton, European Way, Southampton, SO14 3ZH, United Kingdom. suth@noc.soton.ac.uk 


\section{INTRODUCTION}

The Magellan region is geologically young. Although glaciation in the biogeographic Magellan region (sensu Camus 2001) did not reach the extent experienced by Antarctica, an extensive ice cap from 35 to $55^{\circ} \mathrm{S}$ did exist during the Late Glacial Maximum (LGM), approximately 21 ky ago (Benn $\&$ Clapperton 2000). The processes of gradual warming following the LGM explain much of the modern biogeographic pattern in the Magellan region (Arntz et al. 2005). During the glacial period sea level was between ca. 125 and $135 \mathrm{~m}$ lower than it is today (Fairbanks 1989, Yokoyama et al. 2000). The earliest incursions of seawater into the Straits of Magellan occurred by the end of deglaciation around $8 \mathrm{ky}$ ago. It has been proposed that the Straits did not fully open until approximately $7 \mathrm{ky}$ ago (McCulloch \& Davies 2001). All present species in Magellan waters therefore recolonised this region from adjacent Atlantic and Pacific areas (Montiel et al. 2005a). The area is a meeting place for water bodies from the Atlantic and Pacific, and is also partially influenced by the Southern Ocean (Panella et al. 1991).
The modern hydrologic regime in the Straits of Magellan and the Beagle Channel reflects the complex geomorphology and topography, and is highly variable (Brambati et al. 1991, Dávila et al. 2002). The regime is characterised by strong freshwater input from the runoff of high precipitation, sufficient to establish a strong and shallow pycnocline, mean temperature of $7-9^{\circ} \mathrm{C}$ and salinity of 30 (Artegiani \& Pachini 1991, Dávila et al. 2002). High sediment loads are associated with these inputs in regions of glacial action (Brambati et al. 1991). The regime varies significantly between narrows and basins, e.g. currents of $1 \mathrm{~m} \mathrm{~s}^{-1}$ on the Atlantic side of the Straits decrease to $0.2 \mathrm{~m} \mathrm{~s}^{-1}$ in the Paso Ancho (Michelato et al. 1991). This results in characterisation of these zones by coarse and fine sediments respectively (Brambati et al. 1991). Additionally, a wide depth range exists with depths reaching $1200 \mathrm{~m}$ at the western gateway to the Straits (Antezana et al. 1992). These factors have been identified as significant in structuring Magellan invertebrate communities (Montiel et al. 2005b, Moreno et al. 2008).

The aim of this study was to develop the existing characterisation of the macrobenthic ecology

Table 1. Data for sample stations in the Straits of Magellan and the Beagle Channel from the Chilean "Cimar Fiordo 3" expedition in October 1997. For station map see Thatje \& Mutschke (1999a).

\begin{tabular}{|c|c|c|c|c|l|}
\hline Station & Date & Latitude $(\mathbf{S})$ & Longitude $\mathbf{( W )}$ & Depth $\mathbf{( m )}$ & \multicolumn{1}{|c|}{ Location } \\
\hline 2 & $22 / 10$ & 52.32 & 69.20 & 40 & E. Magallanes (Posesión) \\
5 & $22 / 10$ & 53.06 & 70.55 & 51 & E. Magallanes (Paso Ancho) \\
6 & $22 / 10$ & 53.28 & 70.69 & 195 & E. Magallanes (Paso Ancho) \\
8 & $14 / 10$ & 53.86 & 71.53 & 270 & E. Magallanes (Bahía Snug) \\
12 & $12 / 10$ & 52.98 & 73.81 & 571 & E. Magallanes (C. Tamar) \\
13 & $11 / 10$ & 52.78 & 74.20 & 192 & E. Magallanes (Islas Parker) \\
15 & $11 / 10$ & 52.74 & 74.93 & 91 & E. Magallanes (B. Occidental) \\
16 & $11 / 10$ & 53.14 & 73.08 & 411 & G. Xaultegua \\
24 & $13 / 10$ & 53.36 & 71.79 & 130 & E. Silva Palma \\
29 & $14 / 10$ & 54.46 & 70.43 & 162.5 & Seno Agostini \\
30 & $14 / 10$ & 54.41 & 71.03 & 102 & Canal Magdalena \\
41 & $16 / 10$ & 54.90 & 67.57 & 35 & Canal Beagle (Puerto Williams) \\
42 & $17 / 10$ & 55.19 & 66.77 & 45 & Paso Richmond (Isla Lennox) \\
49 & $18 / 10$ & 55.10 & 68.25 & 246 & Bahía Nassau (S. Ponsonby) \\
50 & $18 / 10$ & 55.12 & 68.82 & 65 & Seno Ponsonby \\
51 & $20 / 10$ & 54.58 & 69.33 & 173 & Bahía Parry \\
52 & $20 / 10$ & 54.41 & 69.19 & 112 & Seno Almirantazgo \\
53 & $20 / 10$ & 54.21 & 69.86 & 290 & Seno Almirantazgo \\
54 & $20 / 10$ & 53.88 & 70.26 & 290 & Canal Whiteside \\
55 & $20 / 10$ & 53.61 & 70.27 & 272 & Bahía Inútil \\
56 & $20 / 10$ & 53.54 & 69.91 & 62 & Bahía Inútil \\
67 & $16 / 10$ & 53.46 & 69.51 & 45 & Bahía Inútil \\
& & & &
\end{tabular}


of the Magellan region by examining a high-level taxonomic abundance and biomass data for over the entire region, following a diversity approach. Due to the low number of replicates at each sampling station community analysis was considered inappropriate. Biogeographic affinities of the polychaetes, found as the dominant taxon in the Magellan region, with the Antarctic are discussed.

\section{MATERIAL AND METHODS}

Data collection

Sampling was undertaken on the Chilean "Cimar Fiordo 3" expedition in 1997, from AGOR Vidal Gormaz (Thatje \& Mutschke 1999a). A total of 22 stations were sampled: 18 stations located within the Strait of Magellan, adjacent channels and fjords, and 4 stations located within the eastern part of the Beagle Channel, adjacent channels and fjords (Table 1). Depths of sample stations ranged between 35 and $571 \mathrm{~m}$. Sampling was performed using a Reineck box corer (core area $0.017 \mathrm{~m}^{2}$ ), and 2 or 3 cores were taken at each location. Samples were sieved through $0.5 \mathrm{~mm}$ mesh and preserved in $4 \%$ hexamethylenetetramine-buffered formalin prior to sorting. Animals with calcareous shells were transferred to $70 \%$ ethanol following fixation.

Univariate analysis

Species-level identification was conducted by specialists and abundance (ind. $\mathrm{m}^{-2}$ ) was determined from pooled cores per station. Colonial hydrozoan and bryozoan were only considered as present ( 1 ind. $\mathrm{m}^{-2}$ ) or absent for calculation of abundance values. Abundance data for station 5 were not preserved. Other univariate analyses were calculated from all remaining species-level abundance data using Margalef's d for species richness, Pielou's J for evenness and the Shannon-Wiener H' (based on $\log _{e}$ ) index for diversity. Analysis was implemented using the DIVERSE routine in PRIMER v6 (Plymouth Routines in Multivariate Ecological Research; Clarke and Gorley 2006).

\section{RESULTS}

A total of 173 species/morphotypes were identified across all taxa (Table 2). Low-level taxonomic analysis indicated that Polychaeta dominated abundance (67\%), followed by Arthropoda (17\%), Mollusca (5\%) and Echinodermata (4\%) (Fig. 1a). Polychaeta also dominated biomass (38\%), followed by Echinodermata (23\%), Arthropoda (21\%) and Mollusca (10\%) (Fig. 1b). Average abundance of the Magellan region was 2179 ind. $\mathrm{m}^{-2}$ (range 313 - 10168 ind. $\mathrm{m}^{-2}$ ), and average biomass was $36.8 \mathrm{~g} \mathrm{ww} \mathrm{m}^{-2}$ (range 2.4 - $142.3 \mathrm{~g} \mathrm{ww} \mathrm{m}^{-2}$ ). Polychaete abundance was dominated by the families Cirratulidae (271 ind. $\mathrm{m}^{-2}$ ), Ampharetidae (262 ind. $\mathrm{m}^{-2}$ ), Spionidae (227 ind. $\mathrm{m}^{-2}$ ) and Paraonidae (157 ind. $\mathrm{m}^{-2}$ ) with the most speciose families the Spionidae (6), Lumbrineridae (5), Nereididae (4) and Orbiniidae (4).

Abundance, biomass and species richness all decreased exponentially with depth (Fig. 2a, $\mathrm{b}$ and $\mathrm{c}$ ). Linear regression analysis of natural log transformed abundance and biomass data over log transformed depth indicated that both measures co-varied significantly with natural log transformed

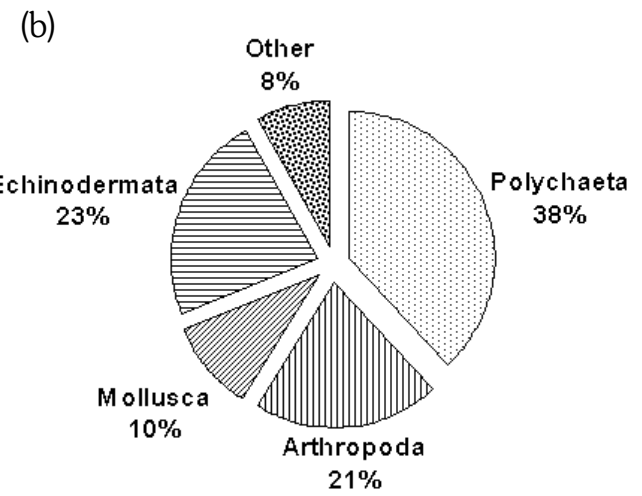

Fig. 1. Relative proportions of taxa $\mathbf{a}$ in macrobenthic abundance and $\mathbf{b}$ in macrobenthic biomass in samples from the Straits of Magellan and the Beagle Channel taken during the Chilean "Cimar Fiordo 3" expedition in 1997. 
TABLE 2. Macrobenthic invertebrates found in samples from the Straits of Magellan and the Beagle Channel taken during the Chilean "Cimar Fiordo 3" expedition in 1997 using a Reineck box corer. The number of individuals per metre squared at each station is shown. Colonial organisms are indicated by $\mathrm{P}=$ presence. Polychaete species known to co-occur in Antarctica are indicated by an asterisk (after Cañete, pers. Communication, Montiel 2005, and references therein).

\begin{tabular}{|c|c|c|c|c|c|c|c|c|c|c|c|c|c|c|c|c|c|c|c|c|c|}
\hline \multirow[t]{2}{*}{ Taxon } & \multicolumn{21}{|c|}{ Station } \\
\hline & 2 & 6 & 8 & 12 & 13 & 15 & 16 & 24 & 29 & 30 & 41 & 42 & 49 & 50 & 51 & 52 & 53 & 54 & 55 & 56 & 57 \\
\hline \multicolumn{22}{|l|}{ PORIFERA } \\
\hline \multicolumn{22}{|l|}{ Demospongiae } \\
\hline Pseudosuberites sp. & 0 & 0 & 0 & 0 & 0 & 0 & 0 & 0 & 0 & 0 & 0 & 0 & 0 & 0 & 0 & 0 & 0 & 0 & 0 & 0 & 42 \\
\hline \multicolumn{22}{|l|}{ CNIDARIA } \\
\hline Hydrozoa & 0 & 0 & 0 & 0 & 0 & 0 & 0 & 0 & 0 & 0 & 0 & 0 & 0 & 0 & 0 & 0 & 0 & 0 & $\mathrm{P}$ & $\mathrm{P}$ & 0 \\
\hline \multicolumn{22}{|l|}{ Anthozoa } \\
\hline Bolocera tuediae subsp. kerguelensis & 0 & 0 & 0 & 0 & 0 & 0 & 0 & 0 & 0 & 21 & 0 & 0 & 0 & 0 & 0 & 0 & 0 & 0 & 0 & 0 & 0 \\
\hline Choriactis laevis & 0 & 0 & 0 & 0 & 0 & 0 & 0 & 0 & 0 & 0 & 0 & 0 & 0 & 0 & 0 & 0 & 0 & 0 & 0 & 21 & 0 \\
\hline NEMERTEA & 729 & 0 & 0 & 42 & 313 & 84 & 0 & 42 & 21 & 0 & 250 & 479 & 0 & 0 & 104 & 83 & 125 & 0 & 0 & 0 & 134 \\
\hline \multicolumn{22}{|l|}{ PRIAPULA } \\
\hline \multicolumn{22}{|l|}{ Priapulida } \\
\hline Priapulopsis tuberculatospinosus & 0 & 0 & 0 & 0 & 0 & 0 & 0 & 0 & 0 & 0 & 0 & 0 & 0 & 0 & 21 & 0 & 0 & 0 & 0 & 0 & 0 \\
\hline \multicolumn{22}{|l|}{ SIPUNCULA } \\
\hline \multicolumn{22}{|l|}{ Sipunculida } \\
\hline Golfingia margaritacea & 0 & 0 & 0 & 0 & 0 & 0 & 0 & 0 & 0 & 0 & 0 & 0 & 0 & 63 & 0 & 0 & 0 & 0 & 0 & 0 & 0 \\
\hline Nephasoma diaphanes & 0 & 21 & 21 & 0 & 83 & 0 & 0 & 0 & 0 & 42 & 21 & 0 & 0 & 0 & 0 & 0 & 0 & 0 & 0 & 0 & 0 \\
\hline Nephasoma sp. & 0 & 0 & 0 & 0 & 0 & 0 & 0 & 0 & 0 & 0 & 21 & 0 & 0 & 0 & 21 & 0 & 0 & 0 & 0 & 0 & 0 \\
\hline Phascolion strombus & 0 & 0 & 0 & 0 & 21 & 0 & 0 & 0 & 0 & 0 & 0 & 0 & 0 & 0 & 0 & 0 & 0 & 0 & 0 & 0 & 0 \\
\hline \multicolumn{22}{|l|}{ ANNELIDA } \\
\hline \multicolumn{22}{|l|}{ Polychaeta } \\
\hline Leitoscoloplos cf. minutus & 0 & 0 & 0 & 0 & 0 & 42 & 0 & 0 & 42 & 0 & 0 & 0 & 0 & 0 & 0 & 0 & 0 & 0 & 0 & 0 & 0 \\
\hline Leitoscoloplos cf. kerguelensis* & 0 & 0 & 0 & 0 & 42 & 0 & 0 & 0 & 0 & 0 & 0 & 0 & 0 & 0 & 0 & 0 & 0 & 0 & 0 & 0 & 0 \\
\hline Phylo felix* & 0 & 0 & 0 & 0 & 42 & 0 & 0 & 0 & 0 & 0 & 0 & 42 & 0 & 0 & 83 & 0 & 0 & 0 & 0 & 0 & 0 \\
\hline Nainereis dendritica & 0 & 0 & 0 & 0 & 0 & 42 & 0 & 0 & 0 & 0 & 0 & 0 & 0 & 0 & 0 & 0 & 0 & 0 & 0 & 0 & 0 \\
\hline Aricidea sp. 1 & 375 & 0 & 0 & 42 & 250 & 0 & 42 & 42 & 83 & 0 & 42 & 937 & 125 & 0 & 83 & 83 & 0 & 42 & 0 & 42 & 354 \\
\hline Cirrophorus sp. & 167 & 0 & 0 & 0 & 0 & 0 & 0 & 0 & 0 & 0 & 0 & 333 & 49 & 0 & 42 & 0 & 0 & 0 & 0 & 0 & 0 \\
\hline Levinsenia sp. & 0 & 83 & 0 & 0 & 42 & 0 & 0 & 0 & 42 & 0 & 0 & 0 & 0 & 0 & 0 & 0 & 42 & 0 & 0 & 0 & 0 \\
\hline Cossura cf. chilensis & 0 & 42 & 42 & 0 & 0 & 0 & 0 & 0 & 0 & 0 & 0 & 0 & 146 & 83 & 0 & 0 & 0 & 0 & 0 & 0 & 83 \\
\hline Cossura cf. heterochaeta & 0 & 0 & 0 & 0 & 42 & 0 & 0 & 0 & 0 & 0 & 0 & 0 & 0 & 0 & 0 & 0 & 0 & 0 & 0 & 0 & 0 \\
\hline Laonice cf. cirrata & 0 & 0 & 42 & 0 & 0 & 0 & 0 & 0 & 0 & 0 & 42 & 0 & 0 & 0 & 0 & 0 & 0 & 0 & 0 & 0 & 0 \\
\hline Minuspio patagonica & 0 & 83 & 42 & 0 & 208 & 0 & 0 & 0 & 512 & 0 & 42 & 42 & 624 & 167 & 146 & 83 & 42 & 42 & 83 & 0 & 42 \\
\hline Dispio uncinata & 0 & 42 & 0 & 0 & 146 & 0 & 0 & 0 & 0 & 0 & 0 & 0 & 0 & 375 & 0 & 0 & 0 & 0 & 0 & 0 & 0 \\
\hline Polydora cf. giardi & 0 & 0 & 0 & 0 & 0 & 0 & 0 & 0 & 0 & 0 & 0 & 1562 & 20 & 83 & 0 & 0 & 0 & 0 & 0 & 0 & 0 \\
\hline Spiophanes sp. & 0 & 0 & 0 & 0 & 42 & 0 & 0 & 0 & 0 & 0 & 0 & 146 & 0 & 0 & 0 & 0 & 0 & 0 & 0 & 0 & 0 \\
\hline Prionospio sp. & 0 & 0 & 0 & 0 & 0 & 0 & 0 & 0 & 0 & 0 & 0 & 83 & 42 & 0 & 0 & 0 & 0 & 0 & 0 & 0 & 0 \\
\hline Tharyx sp. & 1958 & 0 & 83 & 0 & 1667 & 0 & 0 & 42 & 208 & 0 & 0 & 292 & 42 & 417 & 146 & 42 & 0 & 0 & 0 & 156 & 42 \\
\hline Cauleriella sp. & 0 & 0 & 0 & 0 & 42 & 0 & 0 & 0 & 42 & 0 & 0 & 0 & 42 & 0 & 167 & 0 & 0 & 0 & 0 & 42 & 271 \\
\hline Cirriformia sp. & 0 & 0 & 0 & 0 & 0 & 0 & 0 & 0 & 0 & 0 & 0 & 42 & 0 & 0 & 0 & 0 & 0 & 0 & 0 & 0 & 0 \\
\hline Notomastus latericeus & 83 & 0 & 42 & 0 & 0 & 0 & 0 & 0 & 0 & 0 & 0 & 0 & 0 & 0 & 42 & 0 & 146 & 42 & 42 & 0 & 42 \\
\hline Capitella cf. capitata & 0 & 0 & 0 & 0 & 0 & 0 & 0 & 0 & 0 & 0 & 42 & 0 & 0 & 0 & 0 & 0 & 0 & 0 & 0 & 0 & 0 \\
\hline Mediomastus sp. & 0 & 0 & 0 & 42 & 146 & 0 & 0 & 0 & 167 & 0 & 0 & 0 & 0 & 0 & 0 & 42 & 42 & 0 & 0 & 42 & 0 \\
\hline Maldanidae indet. 1 & 333 & 42 & 83 & 0 & 42 & 0 & 0 & 0 & 0 & 0 & 0 & 104 & 42 & 0 & 42 & 0 & 0 & 42 & 0 & 42 & 42 \\
\hline Maldanidae indet. 2 & 0 & 0 & 0 & 0 & 42 & 0 & 0 & 0 & 0 & 0 & 0 & 0 & 42 & 0 & 0 & 42 & 0 & 0 & 0 & 0 & 0 \\
\hline Chaetopterus cf. variopedatus & 0 & 0 & 0 & 0 & 0 & 0 & 0 & 0 & 0 & 0 & 0 & 0 & 0 & 0 & 0 & 0 & 0 & 0 & 0 & 0 & 42 \\
\hline Phyllochaetopterus sp. & 0 & 0 & 0 & 0 & 0 & 0 & 0 & 0 & 0 & 0 & 42 & 0 & 0 & 0 & 0 & 0 & 0 & 0 & 0 & 0 & 0 \\
\hline Hyboscolex sp. & 0 & 0 & 0 & 0 & 0 & 0 & 0 & 0 & 0 & 0 & 42 & 0 & 0 & 0 & 0 & 0 & 0 & 0 & 0 & 0 & 0 \\
\hline Scalibregma cf. inflatum & 0 & 0 & 83 & 0 & 0 & 0 & 0 & 0 & 0 & 0 & 0 & 0 & 0 & 0 & 0 & 0 & 0 & 42 & 0 & 0 & 0 \\
\hline Ophelina syringopige & 0 & 0 & 0 & 42 & 0 & 0 & 0 & 0 & 0 & 0 & 0 & 0 & 0 & 0 & 0 & 0 & 0 & 0 & 0 & 0 & 0 \\
\hline Ophelina cf. scaphigera* & 0 & 0 & 0 & 0 & 0 & 0 & 0 & 42 & 0 & 0 & 0 & 0 & 0 & 0 & 0 & 0 & 0 & 0 & 0 & 0 & 0 \\
\hline
\end{tabular}


TABLE 2 (cont.)

\begin{tabular}{|c|c|c|c|c|c|c|c|c|c|c|c|c|c|c|c|c|c|c|c|c|c|}
\hline \multirow{2}{*}{ Taxon } & \multicolumn{21}{|c|}{ Station } \\
\hline & 2 & 6 & 8 & 12 & 13 & 15 & 16 & 24 & 29 & 30 & 41 & 42 & 49 & 50 & 51 & 52 & 53 & 54 & 55 & 56 & 57 \\
\hline \multicolumn{22}{|l|}{ olychaeta (cont.) } \\
\hline Magelona sp. & 0 & 0 & 0 & 0 & 42 & 42 & 0 & 0 & 42 & 0 & 01 & 1248 & 0 & 0 & 0 & 0 & 0 & 0 & 0 & 0 & \\
\hline Eteone sculpta & 42 & 0 & 42 & 0 & 0 & 0 & 0 & 0 & 0 & 0 & 0 & 0 & 0 & 0 & 0 & 0 & 0 & 0 & 0 & 0 & \\
\hline Anaitides cf. patagonica & 0 & 0 & 0 & 0 & 42 & 0 & 0 & 0 & 0 & 0 & 0 & 0 & 0 & 0 & 0 & 42 & 0 & 0 & 0 & 0 & \\
\hline Steggoa cf. magalae & 0 & 0 & 0 & 0 & 0 & 0 & 0 & 0 & 0 & 0 & 42 & 0 & 0 & 0 & 0 & 0 & 0 & 0 & 0 & 0 & \\
\hline Harmothoinae indet. $1^{*}$ & 0 & 0 & 0 & 0 & 0 & 0 & 0 & 0 & 0 & 42 & 0 & 0 & 0 & 0 & 0 & 0 & 0 & 0 & 0 & 0 & 42 \\
\hline Harmothoinc & 0 & 0 & 0 & 0 & 0 & 0 & 0 & 0 & 0 & 0 & 0 & 0 & 0 & 42 & 0 & 0 & 0 & 0 & 0 & 0 & \\
\hline Leanira quatrefage & 0 & 0 & 0 & 0 & 0 & 0 & 0 & 42 & 0 & 0 & 0 & 0 & 0 & 0 & 0 & 42 & 0 & 0 & 0 & 0 & \\
\hline Pholoe sp. & 0 & 0 & 0 & 0 & 0 & 42 & 0 & 0 & 0 & 0 & 42 & 0 & 0 & 0 & 0 & 0 & 0 & 0 & 0 & 0 & 42 \\
\hline Linopherus sp. & 0 & 0 & 42 & 0 & 42 & 0 & 0 & 0 & 0 & 0 & 0 & 0 & 42 & 0 & 83 & 0 & 0 & 42 & 42 & 0 & \\
\hline Hesionidae indet. & 0 & 0 & 42 & 0 & 0 & 42 & 0 & 0 & 0 & 0 & 0 & 0 & 0 & 0 & 然 & 0 & 42 & 42 & 0 & 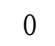 & \\
\hline Ancistrosyllis cf. quellina & 0 & 0 & 0 & 42 & 0 & 0 & 0 & 0 & 0 & 0 & 83 & 42 & 83 & 0 & 83 & 0 & 0 & 0 & 42 & 皮 & \\
\hline Trypanosyllis sp. & 0 & 0 & 42 & 0 & 0 & 0 & 0 & 0 & 0 & 83 & 187 & 0 & 0 & 0 & 0 & 0 & 0 & 0 & 0 & 0 & 42 \\
\hline Typosyllis sp. & 0 & 0 & 0 & 0 & 0 & 0 & 0 & 0 & 0 & 0 & 146 & 83 & 0 & 0 & 0 & 0 & 0 & 0 & 0 & 0 & 0 \\
\hline Langerhansia sp. & 0 & 0 & 0 & 0 & 0 & 0 & 0 & 0 & 0 & 0 & 0 & 0 & 0 & 0 & 0 & 0 & 0 & 83 & 0 & 0 & 0 \\
\hline Syllis s & 0 & 0 & 0 & 0 & 0 & 0 & 0 & 0 & 0 & 42 & 0 & 0 & 0 & 0 & 0 & 0 & 0 & 0 & 0 & & 0 \\
\hline El & 0 & 0 & 0 & 0 & 0 & 0 & 0 & 0 & 0 & 42 & 0 & 0 & 0 & 0 & 0 & 0 & 0 & 0 & 0 & 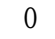 & 0 \\
\hline Exogo: & 42 & 0 & 0 & 0 & 0 & 0 & 0 & 0 & 0 & 0 & 146 & 83 & 0 & 0 & 0 & 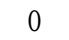 & 0 & 0 & 0 & 0 & 42 \\
\hline Cerato & 42 & 0 & 0 & 0 & 0 & 0 & 42 & 42 & 0 & 0 & 0 & 0 & 104 & 0 & 0 & 0 & 0 & 0 & 0 & 0 & 42 \\
\hline Nereis & 42 & 0 & 0 & 0 & 0 & 0 & & 0 & 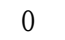 & 0 & 42 & 0 & 0 & 0 & 0 & 0 & 0 & 0 & 0 & 42 & \\
\hline Euner & 0 & 0 & 0 & 125 & 0 & 0 & 4 & 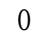 & 0 & 0 & 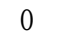 & 0 & 0 & 0 & 0 & 0 & 0 & 0 & 0 & & \\
\hline $\mathrm{Neal}$ & 42 & 0 & 0 & 0 & a & 0 & & 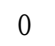 & S & 0 & 42 & 0 & r & 0 & 0 & 0 & 0 & 0 & 0 & & 0 \\
\hline $\mathrm{Hem}$ & 0 & 0 & 42 & 0 & 83 & 83 & 0 & 0 & 0 & 0 & 187 & 125 & 0 & 0 & 0 & 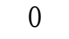 & 0 & 0 & 0 & 42 & 0 \\
\hline Glyc & 83 & 0 & 0 & 0 & 0 & 0 & 0 & 0 & 0 & 0 & 0 & 0 & 0 & 0 & 0 & 42 & 0 & 0 & 0 & 83 & 0 \\
\hline Gonia & 0 & 0 & 0 & 0 & 0 & 0 & 0 & 0 & 0 & 0 & 0 & 0 & 0 & 0 & 0 & 0 & 0 & 0 & 0 & 42 & 0 \\
\hline Oph & 0 & 0 & 0 & 0 & 0 & 0 & 0 & 0 & 0 & 0 & 0 & 42 & 0 & 0 & 0 & 0 & 0 & 0 & 0 & & 0 \\
\hline & 83 & 0 & 0 & 0 & 42 & 0 & 0 & 42 & 0 & 0 & 0 & 42 & 42 & 125 & 42 & 0 & 0 & 0 & 0 & 42 & 83 \\
\hline Nepthys sp.* & 0 & 0 & 42 & 0 & 0 & 0 & 0 & 0 & 0 & 0 & 0 & 0 & 0 & 0 & 0 & 0 & 0 & 0 & 0 & 0 & 0 \\
\hline Nepthys cf. ma & 42 & 0 & 0 & 0 & 0 & 0 & 0 & 0 & 0 & 0 & 0 & 0 & 0 & 0 & 0 & 0 & 0 & 0 & 0 & 0 & 83 \\
\hline Onup & 42 & 0 & 0 & 0 & 83 & 42 & 0 & 0 & 0 & 0 & 0 & 0 & 0 & 0 & 0 & 42 & 0 & 0 & 0 & 0 & 0 \\
\hline Lumb & 0 & 42 & 42 & 42 & 83 & 0 & 0 & 0 & 0 & 0 & 0 & 0 & 0 & 0 & 42 & 0 & 0 & 0 & 0 & & 0 \\
\hline$L u$ & c & 0 & 0 & 0 & 83 & 0 & & 0 & 0 & 0 & 0 & 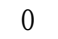 & 0 & 0 & 0 & 0 & 0 & 0 & 0 & 0 & 42 \\
\hline Lumb & 0 & 0 & 0 & 0 & 0 & 0 & 0 & 0 & 0 & 0 & 0 & 83 & 0 & 0 & 0 & 0 & 0 & 0 & 0 & 0 & 0 \\
\hline Lumb & 0 & 42 & 0 & 0 & 0 & 0 & 0 & 0 & 0 & 0 & 0 & 0 & 0 & 0 & 0 & 0 & 0 & 0 & 0 & 0 & 0 \\
\hline Ninoe & 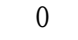 & 42 & 0 & 0 & 42 & 0 & 42 & 83 & 125 & 0 & 42 & 0 & 42 & 83 & 0 & 0 & 83 & 0 & 0 & 0 & 0 \\
\hline Arabell & 0 & 0 & 0 & 0 & 0 & 0 & 0 & 0 & 0 & 0 & 42 & 0 & 0 & 0 & 0 & 0 & 0 & 0 & 0 & 0 & 0 \\
\hline Schist & 83 & 0 & 0 & 0 & 0 & 42 & 0 & 42 & 0 & 0 & 0 & 0 & 0 & 0 & 42 & 0 & 0 & 0 & 0 & 0 & 0 \\
\hline Idan & 0 & 0 & 0 & 0 & 0 & 0 & 0 & 0 & 0 & 0 & 0 & 0 & 0 & 0 & 0 & 0 & 0 & 0 & 0 & 0 & 42 \\
\hline C & 0 & 0 & 0 & 0 & 0 & 0 & 0 & 0 & 0 & 0 & 0 & 42 & 0 & 0 & 42 & 0 & 0 & 0 & 0 & 0 & 0 \\
\hline Flabelligera cf. indura & 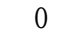 & 0 & 0 & 0 & 0 & - & 0 & 0 & 0 & 0 & 0 & 0 & 0 & 0 & 0 & 0 & 0 & 83 & 0 & 0 & 0 \\
\hline Pherusa sp. & 0 & 0 & 0 & 0 & 0 & 0 & 0 & 0 & 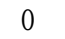 & 0 & 0 & 0 & 0 & 0 & 0 & 0 & 42 & 0 & 0 & 0 & 0 \\
\hline Brada sp. & 0 & 0 & 0 & 42 & 0 & 0 & 0 & 0 & 0 & 0 & 42 & 0 & 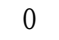 & 0 & 0 & 0 & 0 & 42 & 0 & 0 & 0 \\
\hline Sosanides & 4917 & 42 & 83 & 0 & 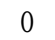 & 0 & & 0 & 42 & 0 & 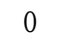 & $0^{2}>$ & 42 & 0 & 0 & 125 & 0 & 83 & 83 & v & 0 \\
\hline & 0 & 0 & $0_{0}>$ & 0 & 0 & 0 & & 0 & 0 & 0 & 83 & 0 & 0 & 0 & 0 & 0 & 0 & 0 & 0 & ( & 0 \\
\hline Tereb & c & 0 & 0 & 0 & 42 & 0 & 42 & 0 & 0 & 0 & 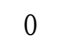 & 0 & 42 & 0 & 42 & 0 & 0 & 0 & 0 & 0 & 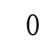 \\
\hline Tricho & 0 & 0 & 0 & 0 & 42 & 0 & 0 & 42 & 0 & 0 & 0 & 0 & 0 & 0 & 0 & 0 & 0 & 0 & 0 & 0 & 0 \\
\hline & c & 0 & 0 & 0 & 0 & 0 & 0 & 0 & 0 & 0 & 0 & 0 & 0 & 0 & 0 & 0 & 0 & 42 & 0 & 0 & 0 \\
\hline Dem & c & 0 & 0 & 0 & 0 & 0 & 0 & 0 & 0 & 0 & 0 & 0 & 0 & 0 & 0 & 0 & 0 & 0 & 0 & 0 & 42 \\
\hline & c & 0 & 0 & 0 & 0 & 42 & 0 & 42 & 0 & 0 & 83 & 0 & 0 & 0 & 0 & 0 & 0 & 0 & 0 & 0 & 0 \\
\hline C & ( & 0 & 42 & 0 & 0 & & 42 & 0 & 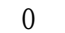 & 0 & 0 & & 0 & 0 & 0 & 0 & 0 & 0 & 0 & 0 & 0 \\
\hline
\end{tabular}


TABLE 2 (cont.)

\begin{tabular}{llllllllllllllllllllll}
\hline Taxon & 2 & 6 & 8 & 12 & 13 & 15 & 16 & 24 & 29 & 30 & 41 & 42 & 49 & 50 & 51 & 52 & 53 & 54 & 55 & 56 & 57 \\
\hline
\end{tabular}

Decapoda

Halicarcinus planatus

Betaeus truncatus

Pagurus comptus

Notiax santarita

\section{Cumacea}

Diastylidae

cf. Ekleptostylis walkeri

Campylaspis sp.

Campylaspis cf. sticta

Leucon meredithi

Eudorella sp. 1

Eudorella sp. 2

\section{Tanaidacea}

\section{Leptochellidae}

Heterotanoides meridionales

Zeuxo phytalensis

Akanthophoreinae

\section{Isopoda}

Platidotea magellanica

Natatolana cf. pastorei

Serolis gaudichaudii

Notasellus chilensis

Munna gallardoi

Munna chilensis

Paramunna cf. integra

Paramunna cf. magellanensis

Pleurosignum cf. elongatum

Rectarcturus kophameli

Jaeropsis intermedius

Anthuridae

\section{Amphipoda}

Ampelisca composita

Ampelisca dentifera

Ampelisca anversensis

Ampelisca sp.

Gondogeneia antarctica

Gondogeneia patagonica

Gondogeneia sp.

Gammaropsis longicornis

Heterophoxus videns

Pseudharpinia sp.

Torometopa sp. 1

Torometopa sp. 2

Stenothoe falklandica

Bathyporeia sp.

Urothoe falcate

Leucothoe spinicarpa

Orchomenella sp.

Orchomene sp.

Tryphosella bispinosa

$\begin{array}{ccccccccccccccccccccc}0 & 21 & 0 & 0 & 0 & 0 & 0 & 0 & 0 & 0 & 0 & 42 & 0 & 0 & 0 & 0 & 0 & 0 & 0 & 0 & 0 \\ 0 & 0 & 0 & 0 & 0 & 21 & 0 & 0 & 0 & 21 & 0 & 0 & 0 & 0 & 0 & 0 & 0 & 0 & 0 & 0 & 0 \\ 0 & 0 & 0 & 0 & 0 & 0 & 0 & 0 & 0 & 0 & 0 & 42 & 0 & 0 & 0 & 0 & 0 & 0 & 0 & 0 & 0 \\ 0 & 0 & 0 & 0 & 0 & 0 & 0 & 0 & 0 & 0 & 21 & 42 & 0 & 0 & 0 & 0 & 0 & 0 & 0 & 0 & 0\end{array}$

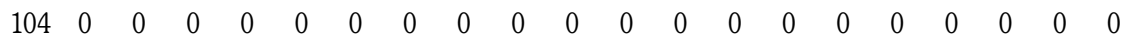

$\begin{array}{lllllllllllllllllllll}0 & 0 & 0 & 0 & 63 & 0 & 0 & 0 & 0 & 0 & 0 & 0 & 0 & 0 & 0 & 0 & 0 & 0 & 0 & 0 & 0\end{array}$

$\begin{array}{lllllllllllllllllllll}0 & 0 & 21 & 0 & 0 & 0 & 0 & 0 & 0 & 0 & 0 & 0 & 0 & 0 & 0 & 0 & 0 & 0 & 0 & 0 & 0\end{array}$

$\begin{array}{lllllllllllllllllllll}0 & 0 & 0 & 0 & 0 & 0 & 0 & 0 & 0 & 0 & 0 & 0 & 0 & 0 & 0 & 42 & 0 & 0 & 0 & 0 & 0\end{array}$

$\begin{array}{lllllllllllllllllllll}0 & 0 & 21 & 0 & 0 & 0 & 0 & 0 & 0 & 0 & 0 & 0 & 0 & 0 & 0 & 0 & 0 & 83 & 0 & 21 & 0\end{array}$

$\begin{array}{lllllllllllllllllllll}0 & 0 & 0 & 0 & 0 & 0 & 0 & 0 & 21 & 0 & 0 & 0 & 0 & 0 & 0 & 0 & 0 & 0 & 0 & 0 & 0\end{array}$

$\begin{array}{lllllllllllllllllllll}0 & 0 & 0 & 0 & 0 & 0 & 0 & 0 & 0 & 21 & 0 & 0 & 42 & 0 & 83 & 0 & 0 & 0 & 0 & 0 & 0\end{array}$

$\begin{array}{lllllllllllllllllllll}21 & 0 & 21 & 0 & 0 & 0 & 0 & 0 & 0 & 0 & 0 & 0 & 0 & 0 & 0 & 0 & 0 & 0 & 0 & 0 & 0\end{array}$

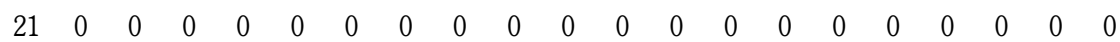
$\begin{array}{lllllllllllllllllllll}0 & 0 & 0 & 0 & 0 & 0 & 0 & 0 & 0 & 0 & 0 & 0 & 0 & 0 & 21 & 0 & 0 & 0 & 0 & 0 & 0\end{array}$ $\begin{array}{lllllllllllllllllllll}0 & 0 & 0 & 0 & 0 & 0 & 0 & 0 & 0 & 0 & 42 & 0 & 0 & 0 & 0 & 0 & 0 & 0 & 0 & 0 & 0\end{array}$

$\begin{array}{lllllllllllllllllllll}42 & 0 & 0 & 0 & 0 & 0 & 0 & 0 & 0 & 0 & 0 & 0 & 0 & 0 & 0 & 0 & 0 & 0 & 0 & 0 & 0\end{array}$ $\begin{array}{lllllllllllllllllllll}0 & 0 & 0 & 0 & 0 & 0 & 0 & 0 & 0 & 0 & 0 & 0 & 0 & 0 & 0 & 0 & 0 & 0 & 21 & 0 & 0\end{array}$ $\begin{array}{lllllllllllllllllllll}21 & 0 & 0 & 0 & 0 & 0 & 0 & 0 & 0 & 0 & 0 & 0 & 0 & 0 & 0 & 0 & 0 & 0 & 0 & 0 & 0\end{array}$ $\begin{array}{lllllllllllllllllllll}0 & 0 & 0 & 0 & 0 & 0 & 0 & 0 & 0 & 21 & 0 & 0 & 0 & 0 & 0 & 0 & 0 & 0 & 0 & 0 & 0\end{array}$ $\begin{array}{lllllllllllllllllllll}0 & 0 & 0 & 0 & 0 & 0 & 0 & 0 & 0 & 0 & 0 & 21 & 0 & 0 & 0 & 0 & 0 & 0 & 0 & 0 & 0\end{array}$

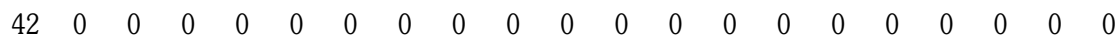
$\begin{array}{lllllllllllllllllllll}0 & 0 & 0 & 0 & 0 & 0 & 0 & 0 & 0 & 0 & 0 & 21 & 0 & 0 & 0 & 0 & 0 & 0 & 0 & 0 & 0\end{array}$ $\begin{array}{lllllllllllllllllllll}0 & 0 & 0 & 0 & 0 & 0 & 0 & 0 & 0 & 0 & 0 & 21 & 0 & 0 & 0 & 0 & 0 & 0 & 0 & 0 & 0\end{array}$ $\begin{array}{lllllllllllllllllllll}0 & 0 & 21 & 0 & 0 & 0 & 0 & 0 & 0 & 0 & 0 & 0 & 0 & 0 & 0 & 0 & 0 & 0 & 0 & 0 & 0\end{array}$ $\begin{array}{lllllllllllllllllllll}0 & 0 & 0 & 0 & 0 & 0 & 0 & 0 & 0 & 0 & 0 & 21 & 0 & 0 & 0 & 0 & 0 & 0 & 0 & 0 & 0\end{array}$ $\begin{array}{lllllllllllllllllllll}0 & 0 & 0 & 0 & 0 & 0 & 0 & 0 & 0 & 0 & 0 & 21 & 0 & 0 & 0 & 0 & 0 & 0 & 0 & 0 & 0\end{array}$ $\begin{array}{lllllllllllllllllllll}0 & 0 & 0 & 0 & 0 & 0 & 0 & 0 & 0 & 0 & 21 & 0 & 0 & 0 & 0 & 0 & 0 & 0 & 0 & 0 & 0\end{array}$

$\begin{array}{lllllllllllllllllllll}0 & 0 & 0 & 0 & 21 & 0 & 0 & 0 & 0 & 0 & 0 & 0 & 0 & 0 & 0 & 42 & 0 & 0 & 0 & 0 & 0\end{array}$ $\begin{array}{lllllllllllllllllllll}42 & 0 & 0 & 0 & 83 & 0 & 0 & 0 & 0 & 0 & 0 & 625 & 0 & 0 & 0 & 0 & 0 & 0 & 0 & 0 & 0\end{array}$ $\begin{array}{lllllllllllllllllllll}83 & 63 & 0 & 0 & 0 & 0 & 0 & 125 & 0 & 0 & 0 & 0 & 0 & 0 & 0 & 0 & 0 & 0 & 0 & 0 & 0\end{array}$

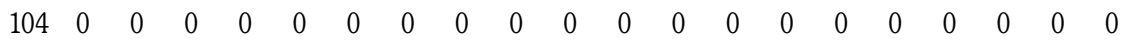
$\begin{array}{lllllllllllllllllllll}0 & 0 & 0 & 0 & 0 & 0 & 42 & 0 & 0 & 0 & 0 & 0 & 0 & 0 & 0 & 0 & 0 & 0 & 0 & 0 & 0\end{array}$ $\begin{array}{lllllllllllllllllllll}0 & 0 & 0 & 0 & 0 & 0 & 0 & 0 & 0 & 0 & 708 & 0 & 0 & 0 & 0 & 0 & 0 & 0 & 0 & 0 & 0\end{array}$ $\begin{array}{lllllllllllllllllllll}0 & 0 & 0 & 0 & 0 & 0 & 83 & 0 & 167 & 0 & 0 & 0 & 0 & 0 & 0 & 0 & 0 & 0 & 0 & 0 & 0\end{array}$ $\begin{array}{lllllllllllllllllllll}83 & 0 & 0 & 0 & 83 & 0 & 0 & 0 & 0 & 0 & 0 & 167 & 0 & 0 & 0 & 0 & 0 & 0 & 0 & 0 & 0\end{array}$ $\begin{array}{lllllllllllllllllllll}0 & 21 & 0 & 0 & 0 & 0 & 0 & 0 & 21 & 0 & 0 & 0 & 0 & 0 & 458 & 375 & 0 & 0 & 0 & 42 & 167\end{array}$ $\begin{array}{lllllllllllllllllllll}0 & 0 & 0 & 0 & 0 & 0 & 0 & 0 & 0 & 0 & 0 & 0 & 21 & 0 & 83 & 0 & 0 & 83 & 0 & 0 & 42\end{array}$ $\begin{array}{lllllllllllllllllllll}0 & 0 & 0 & 0 & 0 & 0 & 0 & 0 & 0 & 208 & 0 & 0 & 0 & 0 & 0 & 0 & 0 & 0 & 0 & 0 & 0\end{array}$ $\begin{array}{lllllllllllllllllllll}0 & 0 & 0 & 0 & 0 & 0 & 0 & 0 & 0 & 83 & 0 & 0 & 0 & 0 & 0 & 0 & 0 & 0 & 0 & 0 & 0\end{array}$ $\begin{array}{lllllllllllllllllllll}0 & 0 & 0 & 0 & 0 & 0 & 0 & 0 & 0 & 0 & 0 & 42 & 0 & 0 & 0 & 0 & 0 & 0 & 0 & 0 & 0\end{array}$ $\begin{array}{lllllllllllllllllllll}0 & 0 & 0 & 0 & 125 & 0 & 0 & 0 & 0 & 0 & 0 & 83 & 0 & 0 & 0 & 0 & 0 & 0 & 0 & 0 & 0\end{array}$ $\begin{array}{lllllllllllllllllllll}0 & 0 & 0 & 0 & 0 & 0 & 0 & 42 & 0 & 0 & 0 & 0 & 21 & 0 & 0 & 0 & 0 & 42 & 0 & 125 & 0\end{array}$ $\begin{array}{lllllllllllllllllllll}0 & 0 & 0 & 0 & 0 & 83 & 0 & 0 & 0 & 0 & 0 & 42 & 0 & 0 & 0 & 0 & 0 & 0 & 0 & 0 & 0\end{array}$

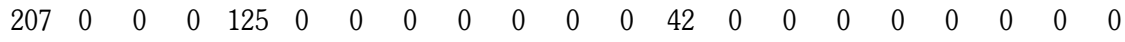
$\begin{array}{llccccccccccccccccccc}0 & 0 & 0 & 0 & 0 & 83 & 0 & 0 & 0 & 0 & 167 & 0 & 0 & 0 & 0 & 0 & 0 & 0 & 0 & 0 & 0\end{array}$ 
TABLE 2 (cont.)

\begin{tabular}{|c|c|c|c|c|c|c|c|c|c|c|c|c|c|c|c|c|c|c|c|c|c|}
\hline \multirow[t]{2}{*}{ Taxon } & \multicolumn{21}{|c|}{ Station } \\
\hline & 2 & 6 & 8 & 12 & 13 & 15 & 16 & 24 & 29 & 30 & 41 & 42 & 49 & 50 & 51 & 52 & 53 & 54 & 55 & 56 & 57 \\
\hline \multicolumn{22}{|l|}{ Amphipoda (cont.) } \\
\hline Tryphosella sp. & 0 & 0 & 0 & 0 & 0 & 0 & 0 & 0 & 0 & 0 & 0 & 42 & 0 & 0 & 0 & 0 & 0 & 0 & 0 & 0 & 0 \\
\hline Atylus sp. & 42 & 0 & 42 & 0 & 0 & 0 & 0 & 0 & 0 & 0 & 0 & 0 & 0 & 0 & 0 & 0 & 0 & 0 & 0 & 0 & 0 \\
\hline Iphimedia magellanica & 0 & 0 & 0 & 0 & 0 & 0 & 0 & 0 & 0 & 0 & 0 & 42 & 0 & 0 & 0 & 0 & 0 & 0 & 0 & 0 & 0 \\
\hline Iphimedia sp. & 0 & 0 & 0 & 0 & 0 & 0 & 0 & 0 & 0 & 0 & 208 & 0 & 0 & 0 & 0 & 0 & 0 & 0 & 0 & 0 & 0 \\
\hline Ostracoda & 104 & 0 & 0 & 0 & 0 & 0 & 0 & 0 & 0 & 187 & 375 & 21 & 0 & 0 & 0 & 0 & 0 & 0 & 0 & 0 & 0 \\
\hline \multicolumn{22}{|l|}{ Cirripedia } \\
\hline Notobalanus flosculus & 0 & 0 & 0 & 0 & 0 & 0 & 0 & 0 & 0 & 125 & 0 & 0 & 0 & 0 & 0 & 0 & 0 & 0 & 0 & 0 & 0 \\
\hline \multicolumn{22}{|l|}{ Pantapoda } \\
\hline species 1 , indet. & 0 & 0 & 0 & 0 & 0 & 0 & 0 & 0 & 0 & 0 & 21 & 0 & 0 & 0 & 0 & 0 & 0 & 42 & 0 & 0 & 0 \\
\hline \multicolumn{22}{|l|}{ MOLLUSCA } \\
\hline \multicolumn{22}{|l|}{ Solenogastrea } \\
\hline Limifossor holopeltatus & 0 & 0 & 0 & 0 & 0 & 0 & 0 & 0 & 0 & 0 & 0 & 0 & 21 & 0 & 0 & 0 & 0 & 0 & 0 & 0 & 0 \\
\hline \multicolumn{22}{|l|}{ Polyplacaphora } \\
\hline species 1 , indet. & 0 & 0 & 21 & 0 & 0 & 0 & 0 & 0 & 0 & 134 & 0 & 0 & 0 & 0 & 0 & 0 & 0 & 83 & 0 & 0 & 0 \\
\hline \multicolumn{22}{|l|}{ Gastropoda } \\
\hline Puncturella cognata & 0 & 0 & 0 & 0 & 0 & 0 & 0 & 0 & 0 & 0 & 21 & 21 & 0 & 0 & 0 & 0 & 0 & 0 & 0 & 0 & 0 \\
\hline Scissurella clathrata & 0 & 0 & 0 & 0 & 0 & 0 & 0 & 0 & 0 & 21 & 0 & 0 & 0 & 0 & 0 & 0 & 0 & 0 & 0 & 0 & 0 \\
\hline Antistreptus magellanicus & 0 & 0 & 0 & 0 & 0 & 0 & 0 & 0 & 0 & 0 & 0 & 0 & 0 & 0 & 0 & 0 & 0 & 0 & 0 & 0 & 83 \\
\hline Cyclostrema sp. & 0 & 0 & 0 & 0 & 0 & 0 & 0 & 0 & 0 & 0 & 21 & 0 & 0 & 0 & 0 & 0 & 0 & 0 & 0 & 0 & 0 \\
\hline Nacella mytilina & 0 & 0 & 0 & 0 & 0 & 0 & 0 & 0 & 0 & 0 & 0 & 0 & 0 & 0 & 0 & 0 & 0 & 0 & 0 & 0 & 42 \\
\hline \multicolumn{22}{|l|}{ Bivalvia } \\
\hline Mytilus chilensis & 0 & 0 & 0 & 0 & 0 & 0 & 0 & 0 & 0 & 0 & 0 & 0 & 0 & 0 & 0 & 0 & 0 & 0 & 0 & 0 & 21 \\
\hline Nucula sp. & 21 & 0 & 0 & 0 & 0 & 0 & 0 & 0 & 0 & 0 & 0 & 21 & 0 & 0 & 83 & 0 & 0 & 0 & 0 & 0 & 0 \\
\hline Ennucula grayi & 0 & 21 & 0 & 0 & 0 & 0 & 42 & 0 & 42 & 0 & 0 & 0 & 0 & 0 & 42 & 0 & 21 & 0 & 0 & 0 & 0 \\
\hline Linucula pisum & 0 & 0 & 0 & 0 & 0 & 0 & 0 & 0 & 0 & 0 & 0 & 0 & 83 & 0 & 0 & 0 & 0 & 0 & 0 & 0 & 0 \\
\hline Limatula pygmaea & 0 & 0 & 0 & 0 & 0 & 21 & 0 & 0 & 0 & 0 & 0 & 0 & 0 & 0 & 0 & 0 & 0 & 0 & 0 & 0 & 0 \\
\hline Chlamys patagonica & 0 & 0 & 0 & 0 & 0 & 0 & 0 & 0 & 0 & 42 & 0 & 0 & 0 & 0 & 0 & 0 & 0 & 0 & 0 & 0 & 0 \\
\hline Cuspidaria patagonica & 0 & 0 & 0 & 0 & 0 & 0 & 0 & 0 & 0 & 0 & 0 & 0 & 0 & 0 & 0 & 0 & 0 & 0 & 0 & 21 & 0 \\
\hline Tawera gayi & 0 & 0 & 21 & 0 & 0 & 0 & 0 & 0 & 0 & 0 & 83 & 0 & 0 & 0 & 0 & 0 & 0 & 0 & 0 & 42 & 0 \\
\hline Thyasira magellanica & 0 & 0 & 0 & 0 & 0 & 0 & 0 & 0 & 0 & 42 & 0 & 0 & 0 & 0 & 0 & 0 & 0 & 0 & 0 & 0 & 0 \\
\hline Thyasira bongraini & 0 & 42 & 0 & 0 & 0 & 0 & 0 & 0 & 0 & 0 & 21 & 0 & 0 & 208 & 0 & 0 & 0 & 0 & 0 & 0 & 0 \\
\hline Mysella sp. & 105 & 0 & 0 & 0 & 0 & 0 & 0 & 0 & 0 & 0 & 83 & 21 & 0 & 0 & 21 & 250 & 0 & 0 & 0 & 83 & 0 \\
\hline Yoldia eightsi & 0 & 0 & 0 & 0 & 0 & 0 & 0 & 0 & 0 & 0 & 0 & 0 & 0 & 0 & 21 & 0 & 0 & 0 & 0 & 0 & 0 \\
\hline Yoldiella valettei & 0 & 0 & 0 & 0 & 0 & 0 & 0 & 0 & 0 & 0 & 0 & 0 & 0 & 0 & 0 & 105 & 0 & 0 & 0 & 21 & 0 \\
\hline Yoldiella cf. granula & 0 & 0 & 0 & 0 & 0 & 0 & 0 & 0 & 0 & 0 & 0 & 21 & 0 & 0 & 0 & 0 & 0 & 0 & 0 & 0 & 0 \\
\hline Propelada longicaudata & 0 & 21 & 0 & 0 & 0 & 0 & 0 & 0 & 0 & 0 & 0 & 0 & 0 & 0 & 0 & 0 & 0 & 0 & 0 & 0 & 0 \\
\hline BRYOZOA & $P$ & $\mathrm{P}$ & $\mathrm{P}$ & 0 & 0 & 0 & 0 & 0 & 0 & $\mathrm{P}$ & $\mathrm{P}$ & 0 & 0 & $P$ & 0 & 0 & 0 & $P$ & 0 & 0 & 0 \\
\hline BRACHIOPODA & 0 & 0 & 0 & 0 & 0 & 21 & 0 & 0 & 0 & 0 & 42 & 0 & 0 & 0 & 0 & 0 & 0 & 0 & 0 & 0 & 0 \\
\hline \multicolumn{22}{|l|}{ ECHINODERMATA } \\
\hline Asteroidea & & & & & & & & & & & & & & & & & & & & & \\
\hline Odontaster meridionalis & 0 & 0 & 0 & 0 & 0 & 0 & 0 & 21 & 0 & 0 & 0 & 0 & 0 & 0 & 0 & 0 & 0 & 0 & 0 & 0 & 0 \\
\hline Ophiuroidea & & & & & & & & & & & & & & & & & & & & & \\
\hline Ophiacantha antarctica & 0 & 0 & 0 & 0 & 21 & 0 & 0 & 0 & 0 & 0 & 0 & 0 & 0 & 0 & 0 & 0 & 0 & 0 & 0 & 0 & 0 \\
\hline Amphiura magellanica & 0 & 0 & 0 & 0 & 0 & 0 & 0 & 0 & 0 & 0 & 0 & 0 & 21 & 0 & 0 & 0 & 0 & 21 & 0 & 0 & 0 \\
\hline Amphiura eugeniae & 0 & 0 & 0 & 0 & 0 & 0 & 0 & 0 & 0 & 907 & 0 & 21 & 0 & 0 & 0 & 0 & 0 & 0 & 0 & 0 & 167 \\
\hline Echinoidea & & & & & & & & & & & & & & & & & & & & & \\
\hline Arbacia dufresnei & 0 & 0 & 0 & 0 & 0 & 0 & 0 & 0 & 0 & 21 & 42 & 21 & 0 & 0 & 0 & 0 & 0 & 0 & 0 & 0 & 0 \\
\hline Pseudechinus magellanicus & 0 & 0 & 0 & 0 & 0 & 0 & 0 & 0 & 0 & 0 & 0 & 0 & 0 & 0 & 0 & 0 & 0 & 0 & 0 & 0 & 21 \\
\hline Holothuroidea & & & & & & & & & & & & & & & & & & & & & \\
\hline Psolus patagonicus & 0 & 0 & 0 & 0 & 0 & 0 & 0 & 0 & 0 & 562 & 0 & 0 & 0 & 0 & 0 & 0 & 0 & 0 & 0 & 0 & 0 \\
\hline Hemioedema spectabilis & 0 & 0 & 0 & 0 & 0 & 0 & 0 & 0 & 0 & 0 & 0 & 0 & 0 & 0 & 0 & 0 & 0 & 0 & 0 & 0 & 42 \\
\hline
\end{tabular}




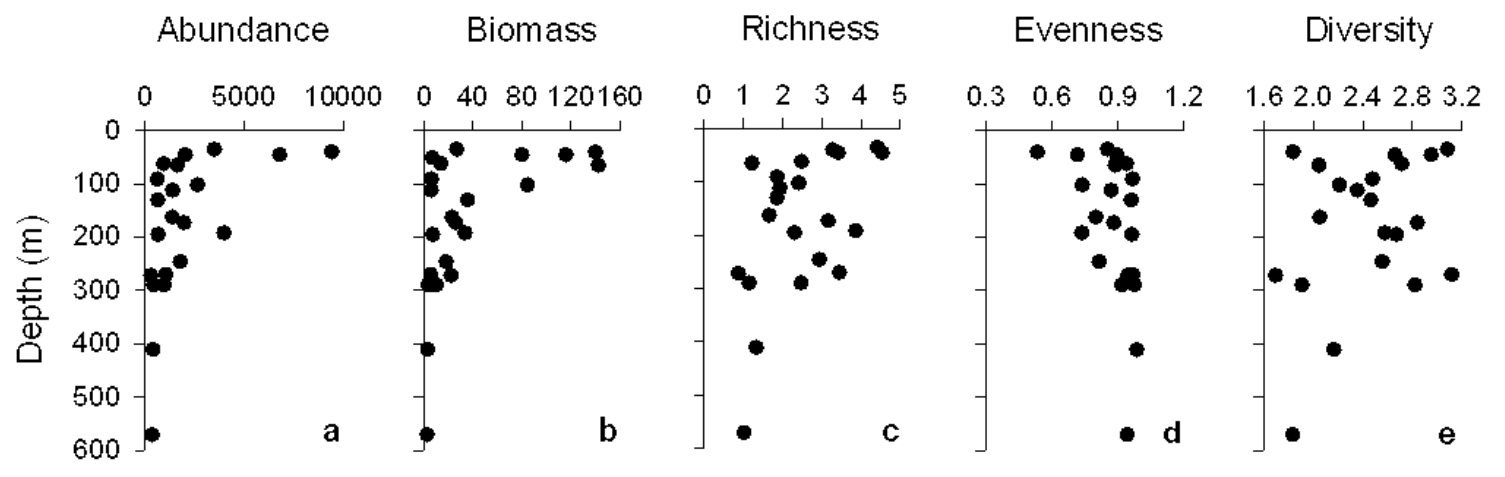

Fig. 2. Univariate analysis of a abundance, $\mathbf{b}$ biomass, $\mathbf{c}$ species richness (Margalef's d), $\mathbf{d}$ evenness (Pielou's J) and $\boldsymbol{e}$ diversity (Shannon Wiener H' based on $\log _{e}$ ) for samples from the Straits of Magellan and the Beagle Channel taken during the Chilean "Cimar Fiordo III" expedition in 1997).

depth $\left(F_{1,19}=17.69, P<0.05\right.$, and $F_{1,20}=14.37 P$ $<0.05$, respectively). Linear regression analysis of species richness data over natural log transformed depth indicated that species richness co-varied significantly with depth $\left(F_{1,19}=7.92, P<0.05\right)$. These analyses yielded $r^{2}$ values that indicated that the natural log depth model explained $48.2 \%$ of the variation in abundance and $41.8 \%$ of the variation in biomass and that the natural log depth model explained $29.4 \%$ of the variation in richness. Evenness increased exponentially with depth (Fig. 2d), and linear regression analysis of evenness data over natural log transformed depth indicated significant co-variation $\left(F_{1,19}=6.13, P<0.05\right)$ with the natural log depth model explaining $24.4 \%$ of the variation in evenness. No trend was observed in diversity with depth (Fig. 2e) and linear regression analysis of diversity data over natural log transformed depth indicated that there was no significant co-variation $\left(F_{1,19}=1.55, P>0.05\right)$. Visual inspection of the residuals for all linear regressions showed approximately homogenous variances and minimal deviation from normality, confirming validity of the test.

\section{DISCUSSION}

Grab sampling has been shown to underestimate epibenthic species that are large, highly motile or rare (Dahm 1996). For example, although large decapod species such as Peltarion spinosulum or motile species such as Munida subrugrosa are known to occur in large numbers in the Magellan region (Gorny 1999, Gutt et al. 1999) they were not found in the present study. A variety of grab cores using the same mechanical principles constitute the dominant benthic sampling method reported for this and comparable regions (Gerdes et al. 1992, Brey \& Gerdes 1999, Gerdes \& Montiel 1999, Thatje \& Mutschke 1999b, Piepenburg et al. 2002) and are therefore the most appropriate method to facilitate contribution to, and comparison with, the existing literature.

This study aimed to assess the macrobenthic ecology through analysis of a large-scale high-level taxonomic abundance and biomass data set. Community analysis was not envisaged for this study with limited sampling undertaken due to time and weather constraints. Despite the low number of stations sampled for the overall area under investigation, clear patterns were established by the diversity analysis. Both the range of abundance and biomass and the means calculated for these parameters strongly resemble other values reported for the Straits of Magellan and the Beagle Channel, and for Antarctica (see Table 2 in Arntz et al. 2005). This is consistent with observations on the absence of latitudinal trends in this region (Brey \& Gerdes 1999, Gerdes \& Montiel 1999, Piepenburg et al. 2002). Similarly, the observed decrease in abundance and biomass with depth is a common pattern and has been reported from numerous other regions, e.g. for the high Antarctic Weddell and Lazarev Seas (Brey \& Gerdes 1998). Pelagic-benthic coupling has been suggested as the dominant factor causing these patterns (Cattaneo-Vietti et al. 1999), with the flux of organic matter from the pelagic to the benthic being the major factor structuring these communities. 
Decreasing species richness and increasing evenness with depth have also been reported from numerous regions, e.g. for the Gulf of Mexico (PérezMendoza et al. 2003). Analysis of these patterns in the polychaetes of the Pacific coast of South America suggests that a source-sink hypothesis of colonisation-extinction dynamics, where shallower "sources" maintain deeper "sinks", provides a conceptual and methodological framework that explains patterns of diversity (Moreno 2008). The absence of any correlating pattern in diversity in the study region is consistent with other studies (Gutt et al. 1999) and may result from variation in hydrologic regime and sedimentation processes between stations and/or the low number of replicates.

Moreno et al. (2006) identified a pattern of decreasing endemism in benthic polychaete species of the Magellan region with increasing latitude. Although species endemic to the Straits of Magellan and the Beagle Channel are therefore less likely to have been observed, the absence of available biogeographic information in the literature prevented an assessment of endemism within the study region. Interestingly, a total of 13 of the 78 polychaete species/morphotypes identified in the samples from this study are known from the Antarctic shelf (e.g. Phylo felix, Leanira quatrefagesi, Nereis eugeniae, Glycinde armata, Idanthyrsus armatus). Clear distribution patterns have been identified between polychaete communities of the Magellan region and Weddell Sea shelves (Montiel et al. 2005b). Polychaete reproductive strategy commonly involves a meroplanktonic larval stage (Giangrande 1997) and Montiel et al. (2005a) suggest that the dispersal of Antarctic species through larval transport in easterly circumpolar currents plays an important part determining in the existing distribution patterns of the fauna around the Magellan region (for discussion see also Thatje \& Fuentes 2003). This is supported by a greater proportion of species with high Antarctic affinities to the Pacific coast compared to the relatively small proportion of species with affinities to the Atlantic side (Montiel et al. 2005a). The durations of larval stages of polychaete species can be extremely short but at lower temperatures have been reported at several months (Bhaud 1998). Reduced densities of larvae observed in the Antarctic and the less obvious seasonality of these larvae may be attributable to further protracted larval development resulting from low temperatures (Stanwell-Smith et al. 1997). However, the key criteria for establishment of a successful population in a new habitat are recruitment conditions and the substrate choice of settling larvae (Raguá-Gil et al. 2004), and therefore the spreading potential of polychaete larvae does not necessarily predict species' adult distribution (Bhaud 1998). Ultimately, given the glacial history of the region, the presence of common species on both sides of the Drake Passage strongly suggests that dispersion is an important process for faunal exchange between the Magellan region and Antarctica, and therefore that for many species the polar front does not necessarily function as a strict barrier (Thatje \& Fuentes 2003, Montiel et al. 2005a).

The macrobenthic ecology identified in this study provides evidence of patterns in the Magellan region that have been reported for numerous other regions, additionally indicating exchange processes by which this region may have been recolonised following glaciation and which may still occur. This is a first study of high taxonomic level analysis providing information that may be of use in future biogeographic studies. Further high resolution sampling is required to develop community analysis of the region and elucidate Magellan-Antarctic connections.

\section{ACKNOWLEDGEMENTS}

The corresponding author would like to thank Erika Mutschke, Carlos Rios, Matthias Gorny, Dieter Gerdes, and Wolf Arntz for supporting his work during Cimar Fiordo 3. We are grateful to the captain and crew of AGOR Vidal Gormaz for assistance at sea. This work would not have been possible without the help in taxonomic identifications by several colleagues (in no particular order): Angelika Brandt, Anja Schmidt, José Saiz-Salinas, Iván Cañete, Claude De Broyer, Martin Rauschert, Ute Mühlenhardt-Siegel, Karin Riemann-Zürneck. We thank Gustavo Lovrich for revising an earlier version. The authors declare that they have no conflict of interest.

\section{LITERATURE CITED}

Antezana, T., L. Guglielmo \& E. Ghirardelli 1992. Microbasins within the Strait of Magellan affecting zooplankton distribution. In: Gallardo, V.A., O. Ferretti, H.I. Moyano (eds) Ocea- 
nografía en Antártica. Santiago, Ediciones Documentadas: 453-458.

Arntz, W.E., S. Thatje, D. Gerdes, J.M. Gili, J. Gutt, U. Jacob, A. Montiel, C. Orejas \& N. Teixidó 2005. The Antarctic-Magellan connection: macrobenthos ecology on the shelf and upper slope, a progress report. Scientia Marina 69 (Suppl. 2):237-269.

Artegiani, A. \& E. Paschini 1991. Hydrological characteristics of the Straits of Magellan: austral summer 1990/91 (February-March 1991). Memorie di Biologia Marina e di Oceanografia 19:77-81.

Benn, D. \& C. Clapperton 2000. Glacial sedimentlandform associations and paleoclimate during the last glaciation, Strait of Magellan. Quaternary Research 54:13-23.

Bhaud, M. 1998. The spreading potential of polychaete larvae does not predict adult distributions: consequences for conditions of recruitment. Hydrobiologia 375-6:35-47.

Brambati, A., C. Fontolan \& U. Simeoni 1991. Recent sediments and sedimentological processes in the Straits of Magellan. Bolletino di Oceanologia Teorica ed Applicata 9(2-3):217-259.

Brey, T. \& D. Gerdes 1998. High Antarctic macrobenthic community production. Journal of Experimental Marine Biology and Ecology 231:191-200.

Brey, T. \& D. Gerdes 1999. Benthic community productivity in the Magellan region and in the Weddell Sea. Scientia Marina 63 (Suppl. 1):145-148.

Camus, P.A. 2001. Biogeografía marina de Chile continental. Revista Chilena de Historia Natural 74:587-617.

Cattaneo-Vietti, R., M. Chiantore, C. Misic, P. Povero \& M. Fabiano 1999. The role of pelagic-benthic coupling at Terra Nova Bay (Ross Sea) and inside the Strait of Magellan. Scientia Marina 63 (Suppl. 1):113-121.

Clarke, K.R. \& R.N. Gorley 2006. PRIMER v6: user manual/tutorial. PRIMER-E, Plymouth, UK.

Dahm, C. 1996. Ecology and population dynamics of Antarctic ophiuroids. Berichte zur Polarforschung 194:1-289.

Dávila, P., D. Figueroa \& E. Müller 2002. Freshwater input into the coastal ocean and its relation with the salinity distribution off austral
Chile (35-55 5 ). Continental Shelf Research 223:521-534.

Fairbanks, R.G. 1989. A 17,000 year glacio-eustatic sea level record: influence of glacial melting rates on Younger Dryas event and deep ocean circulation. Nature 342:637-642.

Gerdes, D. \& A. Montiel 1999. Distributional patterns of macrozoobenthos: a comparison between the Magellan Region and the Weddell Sea (Antarctica). Scientia Marina 63 (Suppl 1):149-154.

Gerdes, D., M. Klages, W.E. Arntz, R.L. Herman, J. Galéron \& S. Hain 1992. Quantitative investigations on macrobenthos communities of the southeastern Weddell Sea shelf based on multibox corer samples. Polar Biology 12:291-301.

Giangrande, A. 1997. Polychaete reproductive patterns, life cycles and histories: an overview. Oceanography and Marine Biology 35:323-386.

Gorny, M. 1999. On the biogeography and ecology of the Southern Ocean decapod fauna. Scientia Marina 63 (Suppl. 1):367-382.

Gutt, J., E. Helsen, W. Arntz \& A. Buschmann 1999. Biodiversity and community structure of the mega-epibenthos in the Magellan region (South America). Scientia Marina 63 (Suppl. 1):155-170.

Michelato, A., E. Accerboni \& P. Berger 1991. Current meter observations in the eastern and central sectors of the Strait of Magellan. Bolletino di Oceanologia Teorica ed Applicata 9(2-3):261-272.

McCulloch, R. \& S. Davies 2001. Late-glacial and Holocene palaeoenvironmental change in the central Strait of Magellan, southern Patagonia. Palaegeography, Palaeoclimatology, Palaeoecology 173:143-173.

Moreno, R.A., C.E. Hernandéz, M.M. Rivadeneira, M.A. Vidal \& N. Rozbaczylo 2006. Patterns of endemism in south-eastern Pacific benthic polychaetes of the Chilean coast. Journal of Biogeography 33:750-759.

Moreno, R.A., M.M. Rivadeneira, C.E. Hernandéz, S. Sampértegui \& N. Rozbaczylo 2008. Do Rapoport's rule, the mid-domain effect or the source-sink hypotheses predict bathymetric patterns of polychaete richness on the Pacific 
coast of South America. Global Ecoology and Biogeography 17:415-423.

Montiel, A. 2005. Biodiversity, zoogeography and ecology of polychaetes from the Magellan region and adjacent areas. Reports on Polar and Marine Research 505:1-112.

Montiel, A., D. Gerdes \& W.E. Arntz 2005a. Distributional patterns of shallow-water polychaetes in the Magellan region: a zoogeographical and ecological synopsis. Scientia Marina 69 (Suppl. 2):123-133.

Montiel, A., D. Gerdes, B. Hilbig \& W.E. Arntz 2005b. Polychaete assemblages on the Magellan and Weddell Sea shelves: comparative ecological evaluation. Marine Ecology Progress Series 297:189-202.

Panella, S., A. Michelato, R. Perdicaro, G. Magazzù, F. Decembrini \& P. Scarazzato 1991. A preliminary contribution to understanding the hydrological characteristics of the Strait of Magellan: Austral Spring 1989. Bolletino di Oceanologia Teorica ed Applicata 9(23):107-126.

Pérez-Mendoza, A., P. Hérnandez-Alcántara \& V. Solis-Weiss 2003. Bathymetric distribution and diversity of deep water polychaetous annelids in the Sigsbee Basin, northwestern Gulf of Mexico. Hydrobiology 496:361-370. Piepenburg, D., M.K. Schmid \& D. Gerdes 2002. The benthos off King George Island (South Shetland Islands, Antarctica): further evidence for a lack of a latitudinal biomass cline in the Southern Ocean. Polar Biology 25:146-158. Raguá-Gil, J.M., J. Gutt, A. Clarke \& W.E. Arntz 2004. Antarctic shallow-water mega-epibenthos: shaped by circumpolar dispersion of local conditions? Marine Biology 114:829839.

Stanwell-Smith D., L.S. Peck, A. Clarke, A.W.A. Murray \& C.D. Todd 1999. The distribution, abundance and seasonality of marine invertebrate larvae in the maritime Antarctic. The Philosophical Transactions of the Royal Society 354:471-484.

Thatje, S. \& E. Mutschke 1999a. Macrofauna bentónica muestreada con un Reineck box corer. Resultados Crucero Cimar-Fiordo 3, Comité Oceanográfico Nacional, Valparaíso, Chile:126-128.

Thatje, S. \& E. Mutschke 1999b. Distribution of abundance, biomass, production and productivity of macrozoobenthos in the sub-Antarctic Magellan Province (South America). Polar Biology 22:31-37.

Thatje, S. \& V. Fuentes 2003. First record of anomuran and brachyuran larvae (Crustacea: Decapoda) from Antarctic waters. Polar Biology 26:270-282.

Yokoyama, Y., K. Lambeck, P. De Dekker, P.J. Johnston \& L.K. Fifield 2000. Timing of the Last Glacial Maximum from observed sea-level minima. Nature 406:713-716. 
S. THATJE \& A. BROWN 